\title{
Das parteipolitische Paradox des Finanzmarktkapitalismus. Aktionärsorientierte Reformen in Deutschland, Frankreich, Italien und den USA*
}

\author{
John W. Cioffi / Martin Höpner
}

An die „Spielarten des Kapitalismus“-Diskussion anknüpfend, überprüft dieser Beitrag die Parteiendifferenzhypothese am Beispiel der gegenwärtigen aktionärsorientierten Transformationen der Unternehmenskontrolle. Aus dem Forschungsstand über die Verteilungswirkungen aktionärsorientierter Unternehmenskontrolle und über die institutionelle Komplementarität zwischen Unternehmenskontrolle und Arbeitsbeziehungen ist die Erwartung abzuleiten, dass das MitteLinks-Spektrum aktionärsorientierten Reformen besonders reserviert gegenüber stehen sollte. Tatsächlich zeigt ein Vergleich von Reformen der vergangenen anderthalb Dekaden das Gegenteil. In Deutschland, Frankreich, Italien und den USA waren die politischen Mitte-Links-Spektren die treibenden Kräfte hinter den aktionärsorientierten Reformen, während Parteien der rechten Mitte die traditionellen Institutionen von organisiertem Kapitalismus, Staatskapitalismus, Familienkapitalismus und Managerialism zu bewahren suchten. Die vergleichende Betrachtung ermöglicht die Benennung von Impulsen, die in systematisch unterschiedlicher Weise auf die jeweiligen politischen Spektren der betrachteten Länder wirkten. Die beobachtete Konfliktkonstellation lässt sich als „Konflikt um Managerherrschaft" modellieren. Wir argumentieren, dass dieser neben den von Klassentheorie und Principal-Agent-Ansatz beschriebenen Grundkonflikten kapitalistischer Gesellschaften eine eigenständige Konfliktdimension repräsentiert, ohne die die Reformen der Unternehmenskontrolle seit den frühen 1990er Jahren nicht zu verstehen sind.

\section{Das parteipolitische Paradox aktionärsorientierter Reformen}

Die Unternehmenskontrolle erweist sich seit den 1990er Jahren als Politikfeld von hoher Dynamik. Der Begriff - als deutsche Übertragung des englischen Fachterminus Corporate Governance - bezeichnet die Gesamtheit der Regeln der Konfliktaustragung zwischen den am Unternehmensgeschehen beteiligten Gruppen, zu denen vor allem Leitungskräfte, Mehrheits- und Minderheitsaktionäre, Betriebsräte, Gewerkschaften und Kreditgeber zählen. Auf technologische Veränderungen auf den Finanzmärkten, das Wachstum institutioneller Investoren und die Internationalisierung ihrer Anlagestrategien, Wettbewerbszunahme, Haushaltsprobleme und demographischen Druck reagieren die Regierungen aller westlichen Industrienationen mit aktionärsorientierten Reformen des Kapitalmarkt- und Unternehmensrechts. Diese Reformen transformieren nicht nur die kontinentaleuropäischen, in der Fachliteratur als „organisiert“ (Winkler

\footnotetext{
* Wir danken den anonymen Gutachtern der PVS für ihre hilfreichen Hinweise.
} 
1974), „rheinisch“ (Albert 1993) oder „koordiniert“ (Hall/Soskice 2001) bezeichneten Spielarten des Kapitalismus und nähern sie dem Idealtyp des liberalen Marktkapitalismus an. Auch der US-amerikanische Kapitalismus ist lediglich in der Theorie ein shareholder capitalism. Amerikanische Unternehmenskontrolle kann als Machtspiel zwischen Managern und Aktionären gedeutet werden, bei dem die dominante Position nicht selten den Führungskräften zugesprochen wurde (Roe 1994). Somit bleibt auch der amerikanische Kapitalismus von aktionärsorientierten Reformen wie dem SarbanesOxley Act von 2002 nicht unberührt.

Die Trennung von Eigentum und Kontrolle über Unternehmen galt Politökonomen unterschiedlichster Richtungen als Ausgangspunkt zur Beschreibung tief greifender Transformationen des modernen Kapitalismus. So wiesen erstmals Berle und Means die historische Übertragung der Macht über die amerikanische Wirtschaft an die Manager nach (Berle/Means 1932/1999; siehe insbesondere auch Burnham 1941) und leiteten daraus die Notwendigkeit öffentlicher Kontrolle über den Unternehmenssektor ab (Berle/Means 1932/1999: 293-313). Die Theoretiker des organisierten Kapitalismus (Hilferding 1927/1982; Naphtali 1928/1967) glaubten ebenso wie Schumpeter (1942), ein Gesetz der abnehmenden Bedeutung des Privateigentums erkannt zu haben, das die Fundamente des Kapitalismus letztlich untergraben müsse. Galbraith (1967) und andere analysierten die wirtschaftlichen und kulturellen Konsequenzen des Managerialism und entwickelten Theorien der modernen „Industriegesellschaft“, die zwar noch kapitalistisch, hinsichtlich ihrer Grundzüge aber von der techno structure beherrscht sei, die Technikanwendung zum Selbstzweck mache, Unternehmen zunehmend Eigenschaften staatlicher Behörden auferlege und dem Wettbewerbsprinzip enge Grenzen setze. Auch Shonfields (1965) vergleichender Arbeit zu den Wesensmerkmalen des „modern capitalism “ lag die Diagnose einer grundlegenden Entfernung vom Eigentümer- und Konkurrenzkapitalismus zugrunde. Konvergenztheoretiker der sechziger und siebziger Jahre des letzten Jahrhunderts wiesen bereits auf immer deutlicher werdende Parallelen zwischen den Politischen Ökonomien der Länder dies- und jenseits des Eisernen Vorhangs hin (Tinbergen 1961; Aron 1964). Kurz: Die Transformation schien nachhaltig, der Konkurrenzkapitalismus obsolet und nicht mehr rückholbar.

Diese Vorhersagen haben sich nicht bewahrheitet. Statt in eine grundlegende Systemtransformation zu münden, setzten spätestens in den 1990er Jahren Gegenbewegungen zu organisiertem Kapitalismus und Managerialism ein. Angesichts effektiveren Schutzes der Minderheitsaktionäre, Märkten für Unternehmenskontrolle, handlungsfähigeren institutionellen Investoren und neuen Instrumenten aktionärsorientierter Unternehmensführung erweist sich die Trennung von Eigentum und Verfügungsgewalt mit den Finanzinteressen der Kapitalmarktteilnehmer als (wieder) vereinbar. Der von Berle und Means, Galbraith, Shonfield und anderen beschriebenen Zurückdrängung urkapitalistischer Prinzipien wird zunehmend die institutionelle Grundlage entzogen. Was an ihre Stelle tritt, ist noch undeutlich. Mit Windolf (2005: 23-25) möchten wir die neue Konfiguration vorläufig als „Finanzmarktkapitalismus" bezeichnen und betonen, dass die Verwendung dieses Begriffs nicht unbedingt institutionelle Konvergenz impliziert. Es existieren unterschiedliche Wege zur Bedienung von Aktionärsinteressen, sodass Raum für unterschiedliche Ausgestaltungen des Finanzmarktkapitalismus ver- 
bleibt. Gleichwohl: mit Managerialismus, techno structure und organisiertem Kapitalismus ist die Aufwertung der Aktionärsinteressen nicht vereinbar.

Die Verschiebungen der Kräfteverhältnisse zugunsten der Investoren wurden in allen westlichen Industrieländern durch politische Reformen flankiert, die vor allem die Börsenaufsicht, den Schutz der Minderheitsaktionäre vor Übervorteilung durch Mehrheitsaktionäre und Kreditgeber, Transparenzauflagen, die unternehmensinternen Kontrollstrukturen und die Regulierung (freundlicher und feindlicher) Übernahmen betrafen. Unser Beitrag diskutiert die parteipolitische Seite dieser Reformen. Wir schlagen eine Erklärung für eine Konstellation vor, die wir als „parteipolitisches Paradox des Finanzmarktkapitalismus" bezeichnen: Für die von uns analysierten Fälle Deutschland, Frankreich, Italien und die USA gilt, dass die treibenden Kräfte hinter den aktionärsorientierten Reformen der Unternehmenskontrolle die Parteien des Mitte-Links-Spektrums waren, während Parteien der rechten Mitte ${ }^{1}$ dazu neigten, die Reformen zu bremsen und Managerialism und organisierten Kapitalismus institutionell zu stützen. Zu einem paradoxen Befund wird dieser Sachverhalt, wenn man uns in der Akzeptanz grundlegender Einsichten der „Varieties of Capitalism“-Diskussion folgt und diese mit Befunden der Parteiendifferenzlehre konfrontiert.

Wir argumentieren, dass vom Mitte-Links-Spektrum, relativ zur rechten Mitte, Vorbehalte gegen aktionärsorientierte Reformen erwartet werden sollten. Der Parteiendifferenzlehre zufolge treten systematische Unterschiede in parteipolitischen Mustern hervor, wenn Staatstätigkeit Verteilungsrelationen verändert (Schmidt 1996, 2002; Wilensky 2002). Eine Prämisse unserer Argumentation lautet, dass sich die im Vergleich zum Mitte-Rechts-Spektrum höhere Affinität der Linksparteien mit abhängig beschäftigten Wählerschichten in den vergangenen Dekaden nicht vollständig eingeebnet hat. Entscheidend ist nun die Einsicht, dass liberaler Marktkapitalismus und koordinierter Kapitalismus (in der Terminologie von Hall und Soskice) den Wohlstand unterschiedlich verteilen (Hall/Soskice 2001: 21). Eine plausible Annahme lautet, dass starker Aktionärseinfluss - im Einklang mit den Zielsetzungen des "Shareholder Value" (Rappaport 1986/1999: 112) - den Spielraum zur Quersubventionierung unterdurchschnittlich rentabler Unternehmenssegmente schmälert und den Ausschüttungsdruck auf freie Cashflows erhöht, was Kapitaleinkommen relativ zu Arbeitseinkommen begünstigt. De Jong (1997: 17-20) hat zur Stützung dieser These im Rahmen einer Analyse von Bilanzdaten großer europäischer Unternehmen überzeugende empirische Befunde vorgelegt. Albert (1993: 205) bezeichnet den „rheinischen Kapitalismus“ wegen der von seinen Institutionen herbeigeführten Verteilungsmuster als „modernized and updated version of Social Democracy".

Auch Überlegungen jenseits der Verteilungswirkungen unterschiedlicher Spielarten der Unternehmenskontrolle stützen die Annahme, dass aktionärsorientierte Reformen dem Mitte-Links-Spektrum besonders fern liegen sollten. Feindliche Übernahmen unterminieren langfristige Absprachen zwischen Unternehmensleitungen und Belegschaften, weil Manager, die im Zuge von Übernahmen von ihren Leitungsfunktionen entbunden werden, für die Handlungen ihrer Nachfolger nicht garantieren können. Aktio-

1 Wir wählen durchgängig diese Begriffe, um zu verdeutlichen, dass sich unsere Argumentation nicht auf die kleineren sozialistischen, kommunistischen sowie rechten Parteien außerhalb der Mitte des Parteienspektrums bezieht. 
närsgewinne bei feindlichen Übernahmen resultieren zu einem erheblichen Teil aus dem Bruch impliziter Verträge (Shleifer/Summers 1988). Während Belegschaftsinteressen bei der Vorbereitung ausgehandelter Fusionen und Übernahmen berücksichtigt werden können, sind feindliche Übernahmen ein definitionsgemäß mitbestimmungsfreier Vorgang.

Dieser Sachverhalt lässt sich allgemeiner formulieren: Verändert sich die institutionelle Verfasstheit einer Sphäre von Produktionsregimen - in unserem Fall, indem sie sich näher dem Idealtyp des liberalen Marktkapitalismus annähert -, dann sollte dies Auswirkungen auch auf andere, benachbarte Sphären haben. In der Politischen Ökonomie wird diese Eigenschaft von Institutionen als institutionelle Komplementarität bezeichnet (Aoki 1994). Der Begriff meint, dass eine Institution A ihre (in unserem Beispiel) marktkorrigierende Funktion nur im Zusammenwirken mit einer anderen Institution B ausüben kann, die ebenfalls auf die Korrektur von Marktergebnissen ausgerichtet ist. Als institutionelle Sphären politischer Ökonomien werden in der „Varieties of Capitalism"-Theorie insbesondere das Wettbewerbsregime, Aus- und Weiterbildung, der Wohlfahrtsstaat und die Arbeitsbeziehungen angesehen. Verfügen die betreffenden Institutionen tatsächlich über die Eigenschaft institutioneller Komplementarität, könnte also eine Vermarktlichung der Unternehmenskontrolle auch die Funktionsweisen von Mitbestimmung, koordinierter Lohnfindung oder statusbezogener Sozialpolitik unterminieren, von denen ihrerseits Verteilungswirkungen ausgehen. Hält diese Annahme, sollten Mitte-Links-Parteien insbesondere in organisierten Ökonomien europäischer Prägung kein Interesse an aktionärsorientierten Reformen haben.

Wir vergleichen das Verhalten der großen Parteien im Zuge der wichtigsten aktionärsorientierten Reformen der vergangenen anderthalb Jahrzehnte in vier Ländern: Deutschland, Frankreich, Italien und den USA. Alle vier Länder zählen zur Gruppe der G8, der bedeutendsten und wohlhabendsten kapitalistischen Industrienationen. Hinsichtlich der institutionellen Verfasstheit der Unternehmenskontrolle repräsentieren die Länder unterschiedliche Modelle. In Frankreich war die Unternehmenskontrolle in hohem Maß politisch koordiniert. Die italienische Unternehmenskontrolle wurde mitunter als „Familienkapitalismus" (z.B. Pagano/Trento 2002: 3) umschrieben; dieser Begriff verweist auf eine - anders als in Frankreich und Deutschland - in geringem Maß formalisierte Koordination zwischen Managern, Aktienbesitz in Familienhand und politischen Eliten. Anhand des deutschen Falls entwickelten Hall und Soskice (2001: 2127) ihren Idealtyp einer koordinierten Ökonomie. Wirtschaftliche Koordination war stark formalisiert und erfolgte, anders als in Italien, unter Einschluss der Organisationen des Faktors Arbeit. Amerikanische Unternehmenskontrolle bewegt sich im Spannungsfeld von Aktionärsorientierung und Managerialism. Die Unterschiedlichkeit der Ausgangssituationen in den betrachteten Ländern könnte also größer kaum sein.

Nicht nur waren die Unternehmenskontrollsysteme in den betrachteten vier Ländern vor den aktionärsorientierten Reformen höchst unterschiedlich ausgestaltet. Auch die Spielarten der Demokratie variierten. Lijphart (1999) hat zur Klassifizierung entwickelter Demokratien die Unterscheidung zweier Dimensionen vorgeschlagen: die Exekutive-Legislative-Dimension sowie die Föderalismus-Unitarismus-Dimension, die von Schmidt (2000: 351-355) zu einer Rangfolge der institutionellen Barrieren gegen die zentralstaatliche Legislative und Exekutive ausgebaut wurde. Trägt man die Exekutive- 
Legislative-Dimension und die Föderalismus-Unitarismus-Dimension in einem Koordinatensystem $\mathrm{ab}$, besetzen die vier betrachteten Länder jeweils ein Feld der sich ergebenden Vier-Felder-Matrix und bilden also die maximal mögliche Varianz ab (Lijphart 1999: 255). Zudem variieren auch die Anzahl der relevanten Parteien und ihre Traditionen und politischen Positionierungen. Die jeweiligen Parteien der linken Mitte, etwa amerikanische Demokraten, deutsche Sozialdemokraten und (ab den frühen 1990er Jahren) italienische Postkommunisten, entstammen ebenso unterschiedlichen Parteienfamilien wie französische UDF und RPR, deutsche Christdemokraten und USamerikanische Republikaner (vergleiche etwa die Klassifizierungen in Huber et al. 1997/2004).

Trotz dieser Unterschiede hinsichtlich Unternehmenskontrolle, politischen Institutionen und Parteiensystemen fördert unsere Analyse eine auffällige Parallele zutage. In allen betrachteten Ländern gingen den Reformen der Unternehmenskontrolle Finanzmarktreformen voraus, bei deren Verabschiedung keine auffälligen parteipolitischen Auseinandersetzungen auftraten. Das änderte sich, sobald die Reformen auf den Bereich der Unternehmenskontrolle ausgedehnt wurden. Ab hier wurden die Reformen Gegenstände des Parteienwettbewerbs, allerdings entgegengesetzt der oben formulierten Erwartung: In Deutschland, Frankreich, Italien und den USA waren jeweils die auf der sozioökonomischen Konfliktachse im Sinne Kitschelts (1994: 20-30) weiter links stehenden Großparteien die treibenden politischen Kräfte hinter den aktionärsorientierten Reformen, während die rechts von ihnen positionierten Volksparteien bremsend auf sie einwirkten. Die Mitte-Links-Parteien näherten die Unternehmenskontrolle damit dem bei Hall und Soskice (2001) beschriebenen Idealtyp der liberalen Marktökonomie (LME) an, obwohl dies der politikökonomischen Literatur zufolge doch eine für den Faktor Arbeit ungünstigere Verteilungsrelation herbeiführen sollte. Wir zeigen nachfolgend, dass diese paradoxe Parteienkonstellation in den vier Ländern tatsächlich existiert. Anschließend bieten wir eine Erklärung für das Parteienparadox und diskutieren Implikationen für die Politische Ökonomie.

\section{Deutschland}

Das deutsche System der Unternehmenskontrolle unterscheidet sich von den französischen, italienischen und amerikanischen Spielarten durch ein hohes Maß an Selbstkoordination unter Einschluss des Faktors Arbeit. Direkte staatliche Interventionen in Investitions- und Finanzierungsentscheidungen nach französischem Vorbild waren für Deutschland untypisch, der Anteil staatlicher Unternehmen im Vergleich zu Frankreich und Italien niedrig. Gleichwohl fand die Selbststeuerung des Unternehmenssektors im Schatten eines interventionsfähigen Staats und damit unter Berücksichtigung öffentlicher Interessen statt (Streeck 2001: 1-5). Im Zentrum des bereits in der Spätphase der Industrialisierung entstandenen Netzwerks aus Kapital- und Personalverflechtungen befanden sich Finanzunternehmen, die ihrerseits horizontal verflochten waren (Hilferding 1909/1923: 220-296; Windolf/Beyer 1995; Beyer 1998) und mit gesamtwirtschaftlicher Perspektive regulierend in den Wettbewerb zwischen Industrieunternehmen eingriffen. Die Großbanken, obgleich (anders als der Sparkassensektor) privatwirtschaftlich 
organisiert, wiesen enge Verbindungen mit staatlichen Stellen auf, die in der Frühphase der Bundesrepublik besonders in den Bereichen der Außenwirtschaftsbeziehungen und der Exportförderung gewachsen waren (Dyson 1986: 125-128). Dieses Arrangement korrespondierte mit im Vergleich zu angloamerikanischen Ländern knapp gehaltenen Rechten der Minderheitsaktionäre, schwachen Transparenzauflagen und unterentwickelten Aktienmärkten. Eine Besonderheit des deutschen Falls ist zudem das annähernd paritätisch mitbestimmte, vom Unternehmensvorstand institutionell separierte Aufsichtsorgan (Jackson 2005).

Ähnlich wie in Frankreich und Italien wurde der politische Wille, den Einfluss der Aktionäre auf das Unternehmensgeschehen zu stärken, vor allem durch allgemeine Erwägungen über die (angebotsseitige) Wettbewerbsfähigkeit angestoßen. Im Verlauf der 1980er Jahre weitete sich die "Standortdebatte“ auch auf die Finanzmärkte aus. Mit dem Übergang der achtziger in die neunziger Jahre des letzten Jahrhunderts setzte eine Serie spektakulärer Unternehmenskrisen ein (Metallgesellschaft, Bremer Vulkan, Klöckner, Schneider), die ebenfalls die Bereitschaft erhöhten, die Verantwortlichkeit der Unternehmensleitungen gegenüber den Anteilseignern auszubauen. Zudem, wenn auch weniger ausgeprägt als in Frankreich und Italien, verstärkten Privatisierungsprogramme den politischen Willen zu aktionärsorientierten Reformen.

Um die Belebung der Finanzmärkte zu fördern, verabschiedete die Regierung Kohl (im Amt von 1982 bis 1998) zwischen 1990 und 1998 drei Finanzmarktförderungsgesetze, die vor allem auf den Schutz der Anleger durch Stärkung der staatlichen Aufsicht abstellten (Lütz 2002: 234-248). Unter anderem wurde Insiderhandel zu Lasten der Kapitalmarktteilnehmer erstmals als Straftatbestand eingeführt. Diese Reformen zielten auf eine Aktivierung der Finanzmärkte unter weitgehender Beibehaltung der Grundlinien der traditionellen Unternehmenskontrolle und zogen, ähnlich wie die 1998 ermöglichte Bilanzierung nach internationalen Standards, keine nennenswerten politischen Konflikte nach sich.

Anders verhielt es sich mit den seit den späten 1990er Jahren durchgeführten Reformen. Auch die Unternehmenskontrolle wurde nunmehr als wettbewerbsrelevante Größe angesehen und geriet ins Blickfeld der Angebotspolitik. Das Gesetz zur Kontrolle und Transparenz im Unternehmensbereich (KonTraG), verabschiedet im März 1998, wurde von parteipolitischen Konflikten begleitet. Das Gesetz erweiterte die Haftung der Mitglieder der Leitungsorgane, ermöglichte Aktienrückkäufe und Aktienoptionsprogramme, verbot Höchst- und Mehrfachstimmrechte auf Hauptversammlungen und stärkte die Rechte der Aufsichtsräte bei der Kontrolle der Vorstände. Ohne die mit dem KonTraG verpflichtend eingeführte „one share, one vote“-Regel wäre beispielsweise die feindliche Übernahme des Mannesmann-Konzerns nicht möglich gewesen. Während der Auseinandersetzung um das KonTraG positionierte sich die SPD an der Seite der FDP und verlangte radikalere Reformschritte, vor allem ein Verbot von Bankenbeteiligungen an Industrieunternehmen ab einer Höhe von fünf Prozent des Aktienbestands (dazu auch Busch 2003: 134-135). Die FDP bedauerte, CDU und CSU nicht zu weiterreichenden Reformschritten bewegt haben zu können. ${ }^{2}$ Die Gewerkschaften unterstützten die sozialdemokratische Position und forderten zudem, die Unternehmen

2 Siehe die Stellungnahmen von Hans Martin Bury und Otto Graf Lambsdorff in der Bundestagsdebatte über das KonTraG am 5.3.1998, Bundestagsdokument 13/220. 
gesetzlich auf die Bilanzierung nach internationalen Standards zu verpflichten (ScheibeLange/Prangenberg 1997; Bolt 2000).

Eine ähnliche Konstellation zeigte sich nach dem Regierungswechsel von 1998 während der Auseinandersetzung um das Wertpapiererwerbs- und Übernahmegesetz (WpÜG) vom Dezember 2001, das vor allem wegen der Regelungen zu den Verteidigungsmöglichkeiten bei feindlichen Übernahmeversuchen umstritten war. Zwar lehnten sowohl SPD als auch CDU/CSU im Juni 2001 eine umstrittene Übernahmerichtlinie im Europäischen Parlament ab, die angegriffenen Managements nur vergleichsweise knappe Verteidigungsmöglichkeiten gelassen hätte (Callaghan/Höpner 2005: 321). In Detailfragen nahm gleichwohl die SPD im Vergleich zur CDU/CSU die stärker aktionärsorientierte Position ein. So forderten CDU und CSU, die Geltungsdauer von Vorratsbeschlüssen der Hauptversammlungen für Abwehrmaßnahmen der Vorstände von 18 auf 36 Monate zu erhöhen. Die SPD lehnte diese Forderung ab, um den unkontrollierten Handlungsspielraum der Führungskräfte zu begrenzen. Auch die Abschaffung der Steuer auf Gewinne bei Beteiligungsveräußerungen, beschlossen im Zuge der Steuerreform des Jahres 2000, rief parteipolitische Konflikte hervor. Zweck dieser Steuerentlastung war eine Beschleunigung der Auflösung des deutschen Unternehmensnetzwerks, das die Entstehung eines aktiven Markts für Unternehmenskontrolle bisher verhindert hatte. Die Kanzlerkandidaten Stoiber und Merkel versprachen in den Wahlkämpfen der Jahre 2002 und 2005, die Körperschaftssteuer auf Beteiligungsverkäufe nach Wahlsiegen wieder einzuführen. ${ }^{3}$ Eine Konstellation, in der die SPD den Vorstellungen der Deutschen Schutzgemeinschaft für Wertpapierbesitz (DSW) näher kam als CDU und CSU, ergab sich darüber hinaus im Vorfeld der Verabschiedung des „Vorstandsvergütungs-Offenlegungsgesetzes“ (VorstOG). Kurz: Im Zuge aller relevanter Gesetzespakete erwies sich die SPD, mehr als CDU und CSU, als Partei der Aktionärsinteressen, während CDU und CSU dazu neigten, die traditionellen Institutionen der koordinierten, den Aktionärseinfluss begrenzenden Unternehmenskontrolle zu verteidigen.

\section{Frankreich}

Im Fall der französischen Unternehmenskontrolle beruhten Koordinationsleistungen, die in Deutschland der Selbstorganisation der Beteiligten anheim gestellt waren, auf direkter staatlicher Intervention (Shonfield 1965: 71-87, 121-150; Cioffi 2005: 119). Nach der Verstaatlichungswelle der frühen 1980er Jahre befanden sich nicht nur die wichtigsten Finanzunternehmen wie Suez, Paribas, Crédit Lyonnais, Société Générale und BNP, sondern auch dreizehn der zwanzig größten Industrieunternehmen in voll-

3 Am Rande sei erwähnt, dass diese Fakten die Versuche des damaligen SPD-Vorsitzenden Franz Müntefering im Sommer 2005, seine Partei rhetorisch als Vorkämpferin gegen feindliche Übernahmen zu positionieren („Heuschrecken-Debatte“), ad absurdum führen. Auch die Zulassung von Hedge-Fonds auf dem heimischen Kapitalmarkt, gegen die sich Münteferings Kritik im Besonderen richtete, erfolgte mit dem Investmentmodernisierungsgesetz (InvG) vom Dezember 2003 ohne Not und - dem typischen Muster von Reformen der Finanzmarktregulierung folgend - im Konsens der Parteien. 
ständigem Staatsbesitz (O'Sullivan 2001: 4-8). Insbesondere Allokation und Distribution von Krediten unterlagen damit latenter staatlicher Kontrolle (Zysman 1983: 112144; Levy 1999: 258-284). Eine französische Besonderheit waren zudem die engen, im Gegensatz zu Italien aber weniger ausschließlich auf das bürgerliche Lager konzentrierten personellen Netzwerke zwischen Politik und Wirtschaft. Leitungsorgane der Unternehmen, staatliche Bürokratie und Parteienapparate rekrutieren ihre Führungskräfte zuvörderst aus dem Kreis der Absolventen der Ecole Nationale d'Administration (ENA) und der Ecole Politechnique. So wurde der konservative Präsident Jacques Chirac (im Amt seit 1995) ebenso an der ENA ausgebildet wie der sozialistische Premierminister Lionel Jospin (im Amt von 1997 bis 2002). Bis in die 1990er Jahre hinein waren die Aktionärsrechte in Frankreich auf niedrigem bis mittlerem Niveau ausgeprägt (Fanto 1998), was mit im Vergleich zu Deutschland und Italien geringfügig entwickelteren - im Vergleich zu angloamerikanischen Standards gleichwohl unterentwickelten - Kapitalmärkten korrespondierte.

Die Reformen der Unternehmenskontrolle waren in Frankreich eng mit der Privatisierungsfrage verknüpft. Zwei frühe Privatisierungswellen in den Jahren 1986/87 und 1993 wurden vor allem von Eduard Balladur (RPR) angestoßen, der während des ersten Privatisierungsschubs Finanzminister (im Amt von 1986 bis 1988), während des zweiten Premierminister (im Amt von 1993 bis 1995) war. Hintergründe waren zunächst ideologische Motive der Abkehr von der Verstaatlichungspolitik der frühen 1980er Jahre, und später zunehmend Unzufriedenheit mit der Wirtschaftskraft der Staatsunternehmen und fiskalischer Konsolidierungsdruck. Nach dem Regierungswechsel des Jahres 1997 steigerte die Regierung Jospin das Tempo der Privatisierung. In den ersten zwei Jahren ihres Bestehens privatisierte sie mehr als alle konservativen Regierungen seit 1986 zusammen (Tiberghien 2006: 178, 207-209). Aus Perspektive der Unternehmenskontrolle interessiert allerdings vor allem die Methode der Privatisierung. Während der ersten beiden Privatisierungswellen waren die konservativen Regierungen bestrebt, privatisierte Unternehmen vor dem Einfluss des Kapitalmarkts zu schützen. Aktienpakete wurden nicht an der Börse platziert, sondern bevorzugt an Unternehmen im Kern des personellen Verflechtungsnetzwerks veräußert. Auf diese Weise entstand ein Netzwerk aus (zunächst) stabilen Kapitalbeteiligungen zum Schutz vor feindlichen Übernahmen (les noyaux durs). Von Reformen der Unternehmenskontrolle waren diese Privatisierungsprogramme nicht begleitet (Tiberghien 2006: 181). Eine Reihe von Finanzmarktreformen seit 1985 stärkten die Börsenaufsicht (Goldman 1992: 232-243) und können als Äquivalente zu den - vergleichsweise harmlosen deutschen Finanzmarktförderungsgesetzen angesehen werden.

Im Unterschied dazu wurden die Privatisierungen unter der Regierung Jospin von Reformen zur Stärkung der Aktionärsrechte begleitet und Aktienpakete gezielt an der Börse - vor allem zum Erwerb durch in- und ausländische institutionelle Investoren platziert, was Kritik des bürgerlichen Lagers hervorrief. Im Jahr 2000 präsentierte Jospin Pläne für weitreichende wirtschaftsliberale Reformen, die auch die Unternehmenskontrolle betrafen (nouvelles régulations économiques, NRE). Sie beruhten zum Teil auf den 1995 vorgelegten „best practice“-Empfehlungen einer Kommission um den Vorstandssprecher der Société Générale, Marc Viénot, die von der damaligen Regierung aber nicht in bindendes Recht umgesetzt wurden. Gegenstände waren vor allem Stär- 
kungen der Rechte der Minderheitsaktionäre, verschärfte Transparenzauflagen und eine Verminderung der erlaubten Anzahl der Direktorenmandate, was auf eine gesetzlich vorgeschriebene personelle Entflechtung hinauslief. Im Zusammenhang mit diesen Wirtschaftsreformen rief besonders die Regulierung von Aktienoptionsprogrammen, ein vor allem in angloamerikanischen Ländern angewandtes Instrument zur Kopplung der Managervergütung an den Aktienkurs, parteipolitische und der Richtung nach entgegen der Intuition verlaufende Auseinandersetzungen hervor (Tiberghien 2006: 212 213). Nachdem die Regierung Juppé (im Amt von 1995 bis 1997) im Jahr 1996 die Steuern auf Einkünfte aus Aktienoptionsprogrammen gezielt erhöht hatte, machte die Regierung Jospin diese Reform im Jahr 1998 zum Teil wieder rückgängig und erlaubte eine für die Führungskräfte besonders vorteilhafte neue Form aktienkursabhängiger Vergütung, die "bons de souscrition de parts de créateurs d'enterprise". Die Auseinandersetzung endete mit einem Kompromiss (Besteuerung mit einer Rate von 26 Prozent) und wurde Teil des NRE-Pakets.

Im Jahr 2005 legte der französische Industrieminister Francois Loos (UMP, im Amt seit 2005) den parteipolitisch umstrittenen Plan vor, ausgewählte französische Unternehmen vor dem Hintergrund der mittlerweile nahezu vollständig erodierten noyaux durs (Morin 2000: 38-41) und eines aktiven Markts für Fusionen und Übernahmen gezielt vor feindlichen Übernahmeversuchen zu schützen und damit gegen den Druck der Aktienmärkte abzuschotten. Auch in einer weiteren aktuellen Debatte finden sich die aktionärsorientierteren Positionen auf der Seite der Sozialistischen Partei: Sie schlägt eine deutliche Ausweitung der Haftung der Vorstände, verbunden mit verstärkten Klagerechten einzelner Aktionäre, vor. Die UMP lehnt diese Forderung ab. Insgesamt zeigt sich in Frankreich eine parteipolitische Konstellation, die mit der deutschen vergleichbar ist. In den vergangenen zehn bis fünfzehn Jahren hatte die große Partei links von der Mitte weitergehende Vorstellungen zur aktionärsorientierten Umgestaltung der Unternehmenskontrolle als das bürgerliche Lager. Zu diesem Schluss gelangt auch Tiberghien (2006: 178), der diesen Umstand - wie wir argumentieren werden, zu Unrecht - auf Besonderheiten des französischen Parteien- und Regierungssystems zurückführt. Wie sich nachfolgend zeigen wird, ist das „Parteienparadox" allerdings in keinem Land des betrachteten Samples so ausgeprägt wie in Italien.

\section{Italien}

Der italienische Fall fällt in mancherlei Hinsicht extremer aus als die anderen. Italien war bis in die 1990er Jahre hinein nicht nur durch einen besonders unterentwickelten Kapitalmarkt und gering ausgeprägte Aktionärsrechte, sondern auch durch eine abnormal große Wertdiskrepanz zwischen großen Aktienpaketen und der Summe ihrer Teile - ein in der Finanzwissenschaft gebräuchliches Maß für die Übervorteilung der Streubesitzanleger durch Großanleger, Banken und Manager (Dyck/Zingales 2002: 45) geprägt. Der französischen Unternehmenskontrolle nicht unähnlich, verfügte Italien über einen hohen Anteil verstaatlichter Unternehmen, ein bankenzentriertes Finanzsystem, enge informelle Netzwerke zwischen Staat und Wirtschaft, ein monistisches Board-System und schwach ausgeprägte Beteiligungsrechte der Beschäftigten (Melis 
2000; Bianco 2001). Unterschiede ergeben sich hinsichtlich der Eliten und ihrer Beziehungen zueinander. Als Eigentümer der entscheidenden Industrieunternehmen dominierten ein knappes Dutzend Familienclans wie die Agnelli-Familie, die untereinander enge Beziehungen unterhielten (der so genannte salotto buono) und in ebenso enger Verbindung zu politischen Persönlichkeiten standen (Pagano/Trento 2002). Die informellen, oft korrupten Verflechtungen zwischen Wirtschaft und Politik konzentrierten sich besonders auf die Christdemokratische Partei (DC), die - von zwei Perioden in den 1980er Jahren abgesehen - alle Premierminister der 51 italienischen Koalitionsregierungen zwischen 1946 und 1992 stellte (Kreppel 1997: 331). Neben überdurchschnittlichen Inflations- und Arbeitslosenraten war die italienische Wirtschaft von notorischer Misswirtschaft der öffentlichen Unternehmen geprägt, die von der politischen Klasse als Instrumente der Ämterpatronage genutzt wurden.

Die italienischen Wirtschaftsreformen der 1990er Jahre sind nur vor dem Hintergrund der europäischen Integration und des in der Folge der tangentopoli-Skandale zusammengebrochenen italienischen Parteiensystems zu verstehen. Der Niedergang der etablierten Großparteien, insbesondere aber der DC, brach die jahrzehntelange Dominanz des bürgerlichen Lagers und führte zu einer Serie von Mitte-Links-Regierungen zwischen 1992 und 2001, die nur von Berlusconis erster Amtszeit im Jahr 1994 unterbrochen wurde. Anders als in den meisten anderen EU-Mitgliedsstaaten rief die Aussicht, die nationale Währung im Zuge der europäischen Integration durch den Euro ersetzen zu können, eine Welle der Euphorie hervor. Zur Haushaltskonsolidierung erschienen groß angelegte Privatisierungsprogramme unumgänglich (Pagano/Trento 2002: 25). Im Unterschied zu den französischen Regierungen während der Privatisierungsschübe von 1986/87 und 1993 lehnten es die italienischen Linksregierungen kategorisch ab, Aktienpakete gezielt bei Großanlegern und Banken zu platzieren und staatliche, so genannte „goldene“ Aktien zum Schutz vor Übernahmen in den privatisierten Unternehmen zu belassen.

Der Verkauf der Anteile ehemaliger Staatsunternehmen an Kleinanleger und institutionelle Investoren war nur in Zusammenhang mit verbessertem Schutz der Minderheitsaktionäre und damit dem Verlust von Privilegien der Großeigentümer denkbar. Nach einer Reihe kleinerer Vorläufergesetze (Pagano/Trento 2002: 25; Aganin/Volpin 2003: 24; Deeg 2005: 186-188) erarbeitete eine Reformkommission unter Vorsitz des Leiters des italienischen Schatzamtes, Mario Draghi, Vorschläge an den Gesetzgeber, die eine strikte Neutralitätspflicht feindlich angegriffener Unternehmensleitungen, erweiterten Schutz der Minderheitsaktionäre, eine Stärkung der internen audit committees und verschärfte Transparenzauflagen vorsahen (Draghi 1998; Bianchi et al. 2001: 3039). Diese Reformen markierten den entscheidenden Bruch mit der traditionellen italienischen Unternehmenskontrolle. Sie wurden im Jahr 1998 von der Regierung unter Ministerpräsident Massimo D'Alema (im Amt von 1998 bis 2001) verabschiedet, der der Partito Democratico della Sinistra (PDS), die Nachfolgepartei der Kommunistischen Partei Italiens (PCI), angehörte. „Our Ex-Communists are the most economically liberal party round here" (The Economist 2001: 6), wurde die parteipolitische Konstellation rund um die Wirtschaftsreformen der späten 1990er Jahren umschrieben. Die Nachfolgeparteien der Christdemokraten kritisierten die Wirtschaftsreformen und machten sich für den Schutz der heimischen Industrie vor ausländischen Aktionären 
stark. So ist auch Italien das einzige Land in der Europäischen Union, in dem sich die Mitte-Links-Parteien im Jahr 2001 nachdrücklich für die liberale Fassung der europäischen Übernahmerichtlinie aussprachen, die bürgerlichen Parteien aber dagegen (Callaghan/Höpner 2005: 321).

Mittlerweile befindet sich Berlusconis Forza Italia (im Amt 2001 bis 2006) auf dem besten Weg, im italienischen Parteiensystem den Platz der alten Democrazia Christiana einzunehmen. Im Vergleich zur DC verhält sich die Forza Italia im Spannungsfeld zwischen Erhalt der traditionellen Strukturen des italienischen Familienkapitalismus und Anpassung an den Finanzmarktkapitalismus ambivalenter. Das Wirtschaftsprogramm der Partei ist vergleichsweise wirtschaftsliberal, gleichzeitig aber ist Silvio Berlusconi selbst aus dem salotto buono hervorgegangen und ihm verbunden. Ein im Jahr 2003 verabschiedetes Nachfolgegesetz zu den Draghi-Reformen von 1998 enttäuschte die Finanzmarktteilnehmer. Giorgio La Malfa, der Vorsitzende der Finanzkommission des italienischen Abgeordnetenhauses, betonte in diesem Zusammenhang die Notwendigkeit, den "nationalen Charakter der italienischen Industrie“" zu erhalten; nach den Draghi-Reformen sei allenfalls noch ein "fine tuning" geboten (NZZ 2002: 31). Bei der Abstimmung über die europäische Übernahmerichtlinie im Juni 2001 stimmte die Forza Italia mit den DC-Nachfolgeparteien gegen den Richtlinienvorschlag.

\section{USA}

Der amerikanische Fall ist grundsätzlich anders gelagert als die drei europäischen, gelten die USA in der vergleichenden politikökonomischen Forschung doch als paradigmatisches Beispiel einer liberalen Marktökonomie (Hall/Soskice 2001: 27-33) und ihre Unternehmenskontrolle als „shareholder model“ (z.B. Jacoby 2005: 41). Der Schutz der Minderheitsaktionäre war bereits in den frühen 1990er Jahren stark ausgeprägt, die Wertdiskrepanz zwischen Aktienpaketen und einzelnen Aktien entsprechend gering und die Marktkapitalisierung höher als anderswo. Gleichwohl ist auch in den USA die Ausrichtung der Unternehmen an den Interessen der Aktionäre fragil, umstritten und Gegenstand historischen Wandels. Theorien der Managerherrschaft - in Abgrenzung zur Aktionärsherrschaft - hatten ihren Ursprung fast immer in der Auseinandersetzung mit der amerikanischen Corporate Governance (Berle/Means 1932/1999; Burnham 1941). So kann das amerikanische Unternehmenskontrollsystem als Machtspiel zwischen der unsichtbaren Hand der (Aktien)märkte und der „sichtbaren Hand der Manager" (Chandler 1977) - unter weitgehender Exklusion des Faktors Arbeit - charakterisiert werden (zusammenfassend Roe 1994).

Die feindliche Übernahmewelle der 1980er Jahre markierte eine historische Transformation vom Managerialism zum Finanzmarktkapitalismus. Neben aktionärsorientierten Restrukturierungen führte die Übernahmewelle aber auch zur Konstruktion neuer Abwehrstrategien der Manager (poison pills) und zu für sie vorteilhaften Arrangements selbst im Fall unfreundlicher Übernahmen (golden parachutes). Die Unternehmensskandale der frühen 2000er Jahre - Enron, Worldcom - offenbarten den unkontrollierten Freiraum, den Führungskräfte auch unter den Bedingungen liquider Kapitalmärkte ge- 
nossen und mündeten in massiven Regulierungsschüben zur besseren Kontrolle der Manager. Der Sarbanes-Oxley Act aus dem Jahr 2001 war die umfassendste Reform der amerikanischen Unternehmenskontrolle seit den 1930er Jahren. Das Gesetzespaket zentralisierte das (traditionell den Gliedstaaten zustehende) amerikanische Unternehmensrecht, weitete die Überwachungsbefugnisse der Börsenaufsicht (Securities and Exchange Commission) aus und verschärfte mit der Installierung des Public Company Accounting Oversight Board (PCAOB) die Kontrolle über die Rechnungslegung, stärkte die interne Kontrolle durch die audit committees und legte Managern, Direktoren und Rechnungsprüfern strengere Pflichten und höhere Strafen bei Verletzungen ihrer Pflichten auf (Cunningham 2003: 952-988).

Entscheidend ist an dieser Stelle, dass auch die Reformen der amerikanischen Unternehmenskontrolle einer politischen Dynamik unterlagen, in der die auf der sozioökonomischen Konfliktachse weiter links stehende große Partei - die Demokratische Partei - im Vergleich zur anderen großen Partei die weitergehenden Vorstellungen zur Reichweite aktionärsorientierter Reformen vertrat. Der Sarbanes-Oxley Act war vor allem ein Erfolg der Demokraten, die die Bush-Administration (im Amt seit 2000) mit dem Vorwurf, den Wünschen der Manager mehr Gewicht zuzusprechen als den Interessen der Anleger, vor sich hertrieben. „Since the collapse of Enron opened a season of tough talk about corporate malfeasance, Democrats have generally advocated tighter oversight of accounting and business, while the administration and Republicans in Congress have emphasized punishing corporate wrongdoers without imposing costly new regulations on business", fasste die New York Times den Sachverhalt zusammen (Stevenson/Oppel 2002: 8).

Die US-amerikanischen Reformen unterscheiden sich von den anderen betrachteten Fällen hinsichtlich der politischen Bedeutung der gewerkschaftlich kontrollierten oder beeinflussten Pensionsfonds, die aktiv für den Sarbanes-Oxley Act stritten. Ähnliches gilt, mit der deutschen Situation vergleichbar, für den amerikanischen Gewerkschaftsdachverband AFL-CIO, der den Demokraten traditionell näher steht als der Republikanischen Partei. Die unternehmenspolitischen und lobbyistischen Aktivitäten der amerikanischen Pensionsfonds sind seit jeher Gegenstand politischer Kontroversen. Mittlerweile betreibt die Bush-Administration gegen den Widerstand von Pensionsfonds, Gewerkschaftsverbänden und Demokratischer Partei die Entlastung der Unternehmen von verschiedenen Auflagen des Sarbanes-Oxley-Acts. Und der kalifornische Gouverneur Arnold Schwarzenegger (im Amt seit 2003) arbeitet an der Zerschlagung und Privatisierung der Pensionsfonds der öffentlich Beschäftigten. Besonders dem in Corporate Governance-Fragen beispielhaft aktiven Pensionsfonds CalPERS werfen die Republikaner vor, Hexenjagden gegen Führungskräfte zu betreiben und damit die Wettbewerbsfähigkeit der betroffenen Unternehmen zu gefährden. Phil Angelides (Demokratische Partei), Schatzmeister des kalifornischen Bundesstaats, hält dem entgegen: „That's why the governor and his right-wing ideologues have targeted the pension funds: not because the funds have strayed, but because they are leading the fight on behalf of ordinary shareholders to put transparency and accountability back into American capitalism" (Angelides 2005: 11). 


\section{Vergleichende Betrachtung: Warum greift die Parteiendifferenzhypothese?}

In Deutschland, Frankreich, Italien und den USA wurden seit Mitte der 1990er Jahre Reformen des Finanzmarkt- und Unternehmensrechts zur Stärkung der Position der Aktionäre verabschiedet. Die Regierungen reagierten auf Reformdruck, der von der Internationalisierung von Produktion und Finanzmärkten, dem Wachstum institutioneller Anleger und der internationalen Diversifizierung ihrer Anlagen, Wachstumsschwäche und Haushaltsproblemen sowie spektakulären Unternehmenskrisen ausging. Diese Reformen unterlagen in allen betrachteten Ländern einer vergleichbaren, unserer Ansicht nach erklärungsbedürftigen politischen Dynamik. Das „parteipolitische Paradox des Finanzmarktkapitalismus" scheint unabhängig von der Ausgestaltung der Unternehmenskontrollsysteme vor den Reformen, von der Zugehörigkeit zu Länderfamilien und „Spielarten des Kapitalismus" sowie von Eigenschaften der politischen Systeme und Parteiensysteme zu greifen. Ausschließen können wir, dass die von uns beobachteten Unterschiede einer Regierungs-Oppositions-Dynamik geschuldet sind: In allen vier Ländern fanden während des beobachteten Zeitraums Regierungswechsel statt, durch die die Parteiendifferenzen nicht eingeebnet wurden. Neben der Varianz zwischen den Ländern steht uns also Varianz innerhalb der Länder zur Analyse zur Verfügung. Die Unterschiede zwischen den parteipolitischen Positionen sind nicht immer stark ausgeprägt. Die Finanzmarktregulierung wird in allen betrachteten Ländern vor allem als technische Angelegenheit angesehen. Anders Reformen der Unternehmenskontrolle, die sich nachhaltig auf die Machtverteilung in den Unternehmen auswirken. Im Vergleich der vier Länder finden wir Impulse, die aktionärsorientierte Reformen für das MitteLinks-Spektrum besonders attraktiv erscheinen lassen, sowie Kräfte, die das MitteRechts-Spektrum dazu veranlassen, solche Reformen zu bremsen.

Erstens: Systematische Unterschiede zwischen den Haltungen der Parteien entstehen, sobald Reformen der Unternehmenskontrolle den Einfluss der Minderheitsaktionäre auf Kosten anderer Gruppen stärken. Der Gesetzgeber kann die Rechte der Streubesitzanleger nicht nachhaltig ausweiten, ohne den Handlungsspielraum der vorher privilegierten Führungskräfte und Mehrheitsaktionäre einzuengen. Keine der im Sample vertretenen Mitte-Rechts-Parteien ist eine „Klassenpartei“. Aber die bürgerlichen Parteien sind organisierten Wirtschaftsinteressen personell und programmatisch verbundener als Mitte-Links-Parteien und deshalb zugänglicher für deren Widerstände. Man denke in diesem Zusammenhang etwa an den deutschen CDU-Wirtschaftsrat (Zohlnhöfer 2001: 42-49), der in der Sozialdemokratie keine Entsprechung hat. Die italienischen Christdemokraten sind das extremste Beispiel für enge Beziehungen zwischen Politik und Führungskräften; von italienischen Verhältnissen weit entfernt, sprechen auch Analysen der Parteispenden deutscher Unternehmen eine deutliche Sprache. Obwohl keine der amerikanischen Volksparteien eine Linkspartei im europäischen Sinne ist, zeigen sich doch auch in den USA klare Unterschiede in Bezug auf die Verbundenheit mit organisierten Wirtschaftsinteressen. Manager fühlen sich in großer Mehrheit den Republikanern verbunden (Burris/Salt 1990: 350-351; Judis/Teixeira 2002: 47-49). Im dicht geknüpften französischen Elitenetzwerk sind die Beziehungen zwischen Politik und Wirtschaft stärker als in den anderen drei Ländern über die großen Parteien verteilt. Wir sehen darin eine Ursache für die im Vergleich zu Italien geringer ausge- 
prägten Unterschiede zwischen den parteipolitischen Positionen zu aktionärsorientierten Reformen.

Münden enge Beziehungen zwischen organisierter Wirtschaft und bürgerlichen Parteien in Patronage und Begünstigung im Parteienwettbewerb, ergeben sich für das Mitte-Links-Spektrum gleichzeitig Anreize, wirtschaftliche Machtkonzentration durch rigide Wettbewerbspolitik oder aktionärsorientierte Reformen zu kontrollieren und zu begrenzen. Die sozialdemokratische Haltung während der deutschen ,siebenjährigen Kartellschlacht" der 1950er Jahre, in der die SPD mit Wirtschaftsminister Ludwig Erhard und gegen den katholischen CDU-Flügel für eine marktschaffende Wettbewerbspolitik stritt, erklärt sich zum Teil über diesen Zusammenhang. ${ }^{4}$

Zweitens: Davon unabhängig, wirken auf das Mitte-Links-Spektrum Impulse, die aktionärsorientierte Reformen attraktiv erscheinen lassen. Dazu zählen spezifische Anreize, die Aktie als Anlageform zu fördern. Im Zuge des Maastricht-Prozesses sahen sich Regierungen vor die Notwendigkeit der Sanierung öffentlicher Haushalte gestellt. Alle Regierungen griffen dabei auf das Instrument der Privatisierung zurück. Um bestehende Machtzusammenballungen nicht zusätzlich zu stärken, entschieden sich die französischen und italienischen Mitte-Links-Regierungen gegen den gezielten Verkauf an Industrie und Banken und für die breite Streuung der Anteile an Kleinanleger, nicht zuletzt abhängig Beschäftigte. Eine Voraussetzung dafür ist ein Mindestmaß an Aktionärsschutz zur Bewahrung vor der Übervorteilung durch Manager, Großaktionäre und Kreditgeber. Die Stärkung der privaten Altersvorsorge hat denselben Effekt. Je mehr Arbeitnehmer ihre Ersparnisse in Aktien anlegen - besonders ausgeprägt in den USA -, umso mehr wird Aktionärsschutz zu einem Parameter arbeitnehmerorientierter Politik. Allerdings zum Nachteil anderer Wirtschaftsinteressen, was eine politische Dynamik in Gang setzt. ${ }^{5}$

Daneben, drittens, wirkt ein weiterer, für das Mitte-Links-Spektrum relevanter Impuls. Aktionärsorientierte Reformen sind für die linke Mitte nicht nur im Hinblick auf Arbeitnehmer in ihrer Rolle als Aktionäre attraktiv. Abhängig Beschäftigte haben kein Interesse an unkontrollierten Handlungsspielräumen der Führungskräfte, in denen Manipulation und Selbstbedienung - Enron, Parmalat - gedeihen. Vor die Wahl gestellt, Manager sich selbst zu überlassen oder den Aktionären Kontrollrechte zuzugestehen, haben sich Gewerkschaften besonders in Deutschland und den USA für die Ausweitung der Kontrolle durch die Aktienmärkte entschieden. Hans-Detlev Küller (DGB) bringt diesen Sachverhalt unter Verweis auf die Neigung der Führungskräfte zu sinnlosen Prestigeinvestitionen auf den Punkt: „Wenn insofern der Shareholder-Value-Ansatz dazu beitragen kann, dieses Risikopotential einzugrenzen, kann dies nur im Arbeitneh-

4 In Höpner (2005) erfolgt eine historisch-genetische Analyse der sozialdemokratischen Programmatik in den Bereichen Unternehmenskontrolle und Wettbewerbspolitik. Dort wird gezeigt, dass Opposition gegen wirtschaftliche Machtkonzentration und gegen Managerherrschaft der entscheidende Anstoß für die vergleichsweise liberale Haltung der Sozialdemokratie in den genannten Politikfeldern war.

5 Dieser Zusammenhang stand während der Auseinandersetzungen über den amerikanischen Sarbanes-Oxley Act im Vordergrund. Ziel der Demokraten sei es, so formulierte ihr kalifornischer Repräsentant George Miller, im Sinne der Beschäftigten darauf hinzuwirken „that their savings should not be more vulnerable than those of the people in the big offices and the corner suites" (Labaton/Clymer 2002). 
merinteresse sein" (Küller 1997: 529). Mehr noch: Aktionärsorientierte Reformen gingen in allen betrachteten Ländern mit Ausweitungen der internen Kontrollrechte gegenüber den Vorständen bzw. den executive directors einher, was im deutschen Fall letztlich eine Stärkung der Mitbestimmung bewirkte. Als das deutsche Mitbestimmungsgesetz im Jahr 1976 verabschiedet wurde, reagierten viele Unternehmen mit einer gezielten Schwächung der Aufsichtsräte, indem sie Kürzungen der Kataloge zustimmungspflichtiger Geschäfte vornahmen (Ulmer 1980: 18). Derzeit gewinnen diese Kataloge, angestoßen vor allem durch die neue Aktienkultur, wieder an Bedeutung. ${ }^{6}$ Weil aber nicht der Vorstand, sondern das Aufsichtsorgan, mitbestimmt, gilt: Jede Stärkung des Aufsichtsrats ist eine Stärkung der Mitbestimmung. ${ }^{7}$ In den USA, obgleich weit von mitbestimmten Leitungsorganen deutscher Prägung entfernt, führte ein paralleler Mechanismus zur Forderung nach Stärkung der Aktionärsrechte durch den Gewerkschaftsdachverband AFL-CIO: Nicht die Aufsichtsorgane, sondern entscheidende Anlagefonds unterliegen Gewerkschaftseinfluss, dessen Wirksamkeit sich im Zuge zunehmender Kontrollrechte der Fonds ausweitet.

Unsere Erklärung des parteipolitischen Paradox des Finanzmarktkapitalismus beruht also nicht darauf, dass das klassische Arbeitnehmermilieu in allen westlichen Industrieländern seit mehreren Dekaden schrumpft, mit ihm allein keine Wahlen mehr zu gewinnen sind und sich linke Parteien deshalb auf der Suche nach neuen Mehrheiten zunehmend in die Mitte bewegen (Piazza 2001). Diese Beobachtung ist zwar korrekt, erklärt aber nicht, warum Mitte-Links-Parteien aktionärsorientierter handeln als ihre Nachbarn zur Rechten des Parteienspektrums. Unser Punkt ist auch nicht, dass die Parteien unseres Ländersamples keine „Klassenparteien“ sind und die Bedeutung der Schichtzugehörigkeit für Wahlentscheidungen tendenziell, in keinem anderen Land so deutlich wie in den USA, rückläufig ist (Dalton 2002: 147-154). Im Gegenteil ist unabdingbarer Bestandteil unserer Argumentation, dass die Verbundenheit mit Arbeitnehmerinteressen nach wie vor unterschiedlich über die großen Parteien der betrachteten Länder verteilt ist. Wir interpretieren die aktionärsorientierte Haltung der Mitte-LinksParteien auch nicht als Ergebnis einer Nixon goes to China-Konstellation, bei der zwar signifikante Präferenzunterschiede zwischen den Parteien bestehen, unpopuläre, aber notwendige Maßnahmen jedoch nur durch die Parteien umsetzbar sind, denen die betreffenden Maßnahmen programmatisch ferner liegen als den Konkurrenzparteien (Ross 2000: 158-165). Wir interpretieren die Neigung der Mitte-Links-Parteien hingegen als Ausdruck der „natürlichen“, im Sinne von Hall (2005) vorstrategischen Präferenzen der Mitte-Links-Parteien.

6 So sieht der Kodex der Regierungskommission Corporate Governance in Ziffer 3.3 vor, dass für Geschäfte von grundlegender Bedeutung Zustimmungsvorbehalte zugunsten der Aufsichtsräte in den Satzungen zu verankern sind. Derzeit wird diskutiert (und von den Gewerkschaften nachdrücklich eingefordert), solche Zustimmungsvorbehalte nach österreichischem Vorbild gesetzlich bindend festzuschreiben.

7 Ähnliches gilt für die für skandinavische Länder typische Mitbestimmung im eingliedrigen Verwaltungsrat. Dort nehmen die Arbeitnehmervertreter Aufgaben wahr, die mit denen der non-executives vergleichbar sind. Werden die Kontrollrechte der non-executives gegenüber den executive directors in mitbestimmten Leitungsorganen ausgeweitet, resultiert dies im Endeffekt in einer Stärkung der Mitbestimmung. 


\section{Diskussion: Implikationen}

Das Politikfeld der Unternehmenskontrolle wurde in der politikwissenschaftlichen Forschung bisher noch nicht in Bezug auf die Parteiendifferenzhypothese untersucht. ${ }^{8}$ Unsere Betrachtung zeigt: Es macht für Reformen der Unternehmenskontrolle einen Unterschied, wer regiert. Obwohl in der Literatur als komplementäre institutionelle Sphären behandelt, scheinen für Unternehmenskontrolle und Wettbewerbspolitik grundsätzlich andere politische Logiken zu greifen als für Arbeitsbeziehungen und Sozialpolitik. In den von uns analysierten Ländern Deutschland, Frankreich, Italien und den USA waren die im Zweifel weiter links stehenden großen Volksparteien die treibenden Kräfte hinter den aktionärsorientierten Reformen der Unternehmenskontrolle und damit hinter der Auflösung dessen, was als „rheinisch“, „organisiert“ oder „koordiniert“ beschrieben wurde.

Reformen der Unternehmenskontrolle fanden seit den 1990er Jahren in ausnahmslos allen westlichen Industrieländern statt. Lassen sich unsere Befunde zu den parteipolitischen Dynamiken hinter diesen Reformen über die von uns betrachteten vier G8Länder hinaus verallgemeinern? Wir erheben nicht den Anspruch einer solchen Verallgemeinerbarkeit. Allerdings haben wir unsere Fälle mit Bedacht so gewählt, dass sich eine Reihe von Hypothesen über länderspezifische Ursachen des „parteipolitischen Paradox" ausschließen lassen. Die Präferenzen der politischen Mitte-Links-Spektren für aktionärsorientierte Reformen beruhen nicht auf der Zugehörigkeit zu einer spezifischen Spielart des Kapitalismus, zu einem Demokratietyp im Sinne Lijpharts (auf beiden von Lijphart unterschiedenen Dimensionen) oder Ausprägungen der jeweiligen Parteiensysteme. Auch das Vorliegen einer Regierungs-Oppositions-Dynamik, die für den beobachteten Parteieneffekt zuständig wäre, kann ausgeschlossen werden.

Mindestens für Deutschland, Frankreich, Italien und die USA gilt: Die Parteiendifferenzhypothese greift. Sie greift allerdings entgegengesetzt der Erwartungen, die unserem Erachten nach zu Recht aus der bestehenden politökonomischen Literatur abzuleiten sind. Analysiert man die Verteilungswirkungen auf einer bipolaren Konfliktachse zwischen Kapital (Eigentümer plus Manager) und Arbeit - und nimmt an, dass aktionärsorientierte Reformen die Verteilungsposition des Faktors Kapital stärken -, erscheint die Annahme, von den Interessenvertretungen der Beschäftigten sei Unterstützung für solche Reformen zu erwarten, abwegig. Für die ökonomische Principal-AgentTheorie (Jensen/Meckling 1976), die danach fragt, wie die Outsider (Aktionäre) die Insider (Manager plus Beschäftigte) dazu bringen können, in ihrem Sinne zu handeln, gilt dasselbe: Die Erwartung, Teile der Insider sollten für die Stärkung der Outsider kämpfen, erscheint nicht realistisch (zum Stellenwert der Principal-Agent-Theorie für die Corporate Governance-Debatte vgl. Cioffi 2000).

8 Eine Theorie der Unternehmenskontrolle, bei der politische Unterschiede entlang der LinksRechts-Achse von Bedeutung sind, stammt von dem Juristen Mark J. Roe (2003: 21-26, 4762). Seine Argumentation ist den Principal-Agent-Theorien zuzurechnen. In Roes Modell sind Insider stets Verursacher von Agency-Kosten und Gegner von deren Senkung; die Möglichkeit, dass Arbeitnehmer und die ihnen nahe stehenden Organisationen politisch an der Senkung der von den Führungskräften ausgehenden Agency-Kosten mitwirken könnten, ist in dem Modell nicht vorgesehen. 
Die politischen Dynamiken der aktionärsorientierten Reformen der vergangenen Jahre sind nur zu verstehen, wenn man erstens innerhalb des Faktors „Kapital“ weiter differenziert, zweitens allen drei hier interessierenden Stakeholder-Gruppen - Aktionäre, Manager, Beschäftigte - eigenständige Interessen zuschreibt und drittens die theoretische Möglichkeit von Koalitionen von jeweils zwei der beteiligten Gruppen gegen die jeweils dritte in das Modell einbezieht (Abbildung 1). Wir gehen der Einfachheit halber von Streubesitzunternehmen ohne Großaktionäre aus. ${ }^{9}$ Es entstehen drei Konfliktlinien: Insider-Outsider-Konflikte, bei denen Manager und Beschäftigte eine Koalition gegen die Aktionäre bilden; Klassenkonflikte, die zwischen Beschäftigten einerseits, Aktionären und Managern andererseits verlaufen; und Konflikte um Managerherrschaft, bei denen Aktionäre und Beschäftigte gegen die Manager koalieren. Wir argumentieren, dass alle drei Konfliktlinien in der Praxis von Bedeutung sind. Unsere Analyse hat aber gezeigt, dass die aktionärsorientierten Reformen der vergangenen Jahre von den Interessengruppen der Beschäftigten und den ihnen nahe stehenden Parteien zu (mitunter verblüffend) weiten Teilen als Konflikte um Managerherrschaft perzipiert wurden - der einzigen Konfliktachse, auf der sich die Beschäftigten auf der Gewinnerseite wähnen, wenn der Aktionärseinfluss steigt. Während der Klassenkonflikt von den Machtressourcentheorien im weiteren und Klassentheorien im engeren Sinne als vorherrschender Konflikt in kapitalistischen Ökonomien analysiert wurde und Insider-Outsider-Konflikte im Zentrum der Principal-Agent-Analyse stehen, wurde dem Konflikt um Managerherrschaft in der theoretischen Literatur bisher keine Beachtung geschenkt. ${ }^{10}$ Diese Konfliktachse ist zum Verständnis der Wirtschaftsreformen der vergangenen anderthalb Dekaden aber unverzichtbar. Weder Klassentheorie noch Principal-Agent-Ansatz halten ein Instrument zur Analyse der beobachteten Konfliktkonstellation bereit.

Gleichwohl löst sich das „parteipolitische Paradox des Finanzmarktkapitalismus“ nicht in Wohlgefallen auf. In sich stimmige und allgemein akzeptierte Theorien der Politischen Ökonomie gehen davon aus, dass sich die Verteilungsrelationen des Faktors Arbeit langfristig verschlechtern, wenn die Macht der Aktienmärkte über die Unternehmen zunimmt und sich das Produktionsregime damit der liberal market economy (LME) annähert (de Jong 1997: 17-20; Hall/Soskice 2001: 21). Nehmen wir für den Moment an, dass Reformen der Unternehmenskontrolle vor allem den Verteilungskonflikt zwischen Managern und Aktionären tangieren. Auf die Verteilungsrelation zwischen Kapital und Arbeit hätten Kräfteverschiebungen innerhalb des Faktors Kapital keine direkten Auswirkungen; sie wären dann eine Frage der Institutionen der Arbeitsbeziehungen, in denen der Klassenkonflikt ausgetragen wird. Indirekte Auswirkungen

9 Im Fall des Vorliegens konzentrierter Eigentümerstrukturen wären die Großaktionäre als eigenständige Kraft einzufügen oder aber - was an dieser Stelle ausreicht, um unser Argument zu verdeutlichen - den Managern zuzurechnen. Entscheidend ist, dass es sich bei den Aktionären in unserem Modell um institutionelle Anleger oder Privataktionäre, nicht um Großaktionäre mit Kontrollpaketen handelt.

10 Jüngst haben Gourevitch und Shinn (2005: 205-276) unseren erstmals in Höpner (2001: 27-33, 51, 2003: 150-200) dargelegten Vorschlag aufgegriffen und politische Koalitionen zwischen Aktionären und Beschäftigten als „transparency coalitions" modelliert. Wir halten diese Bezeichnung für unglücklich, weil die partiellen Interessenüberschneidungen weit über den Sachverhalt der Transparenz hinausreichen. 
Abbildung 1: Drei Koalitionen und Konflikte zwischen Aktionären, Management und Beschäftigten

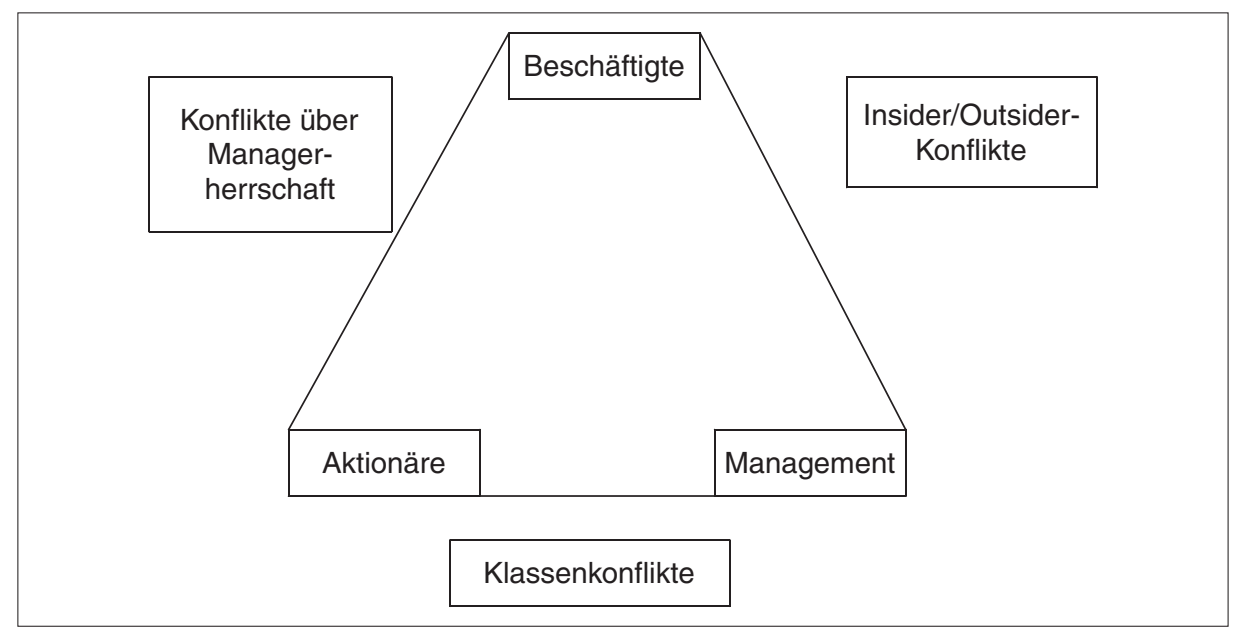

Quelle: Eigene Darstellung.

Angaben: Beispiel: Bei Insider-Outsider-Konflikten bilden Managements und Beschäftigte eine Koalition gegen die Aktionäre.

ergeben sich allerdings auch unter dieser (fragwürdigen) Annahme über die Eigenschaft der institutionellen Komplementarität zwischen Unternehmenskontrolle und Arbeitsbeziehungen, die beispielsweise von Hall und Gingerich (2004: 22-29) überzeugend empirisch nachgewiesen wurde. Demnach darf angenommen werden, dass koordinierte Unternehmenskontrolle - im Sinne von langfristiger, strategischer Interaktion zwischen Managements und Großaktionären unter Abwesenheit aktiver Kapital- und Übernahmemärkte - stabilisierend auf die langfristige Koordination mit den Kernbelegschaften einwirkt (Mitbestimmung, koordinierte Lohnfindung), die ihrerseits eine Machtressource der Gewerkschaften darstellen.

Die Theorie der institutionellen Komplementarität, angewendet auf Arbeitsbeziehungen und Unternehmenskontrolle, scheint nicht zum Verhalten der politischen Mitte-Links-Spektren und, mindestens in Deutschland und den USA, der Gewerkschaften zu passen. Liegt das an kurzsichtigem Handeln der Parteien und Gewerkschaften, oder aber an Fehleinschätzungen der politikökonomischen Forschung? Fest steht, dass die Mitte-Links-Parteien der betrachteten Länder nicht an die Komplementaritäts-These glauben. In Deutschland gilt das auch für die Gewerkschaften. Ihr Einwirken auf die Reformen der Unternehmenskontrolle der vergangenen Jahre zielt auf einen mitbestimmten Finanzmarktkapitalismus, ein Arrangement, das aus Sicht der „Varieties of Capitalism"-Theorie nicht vielversprechend, weil inkohärent und vielleicht wegen der Inkompatibilität seiner Bestandteile nicht einmal möglich erscheint. Zumindest der These der Inkompatibilität ist aber entgegen zu halten, dass der mitbestimmte Finanzmarktkapitalismus aus Sicht der großen Streubesitzunternehmen bereits greifbar nahe ist (zur Kompatibilität von Aktionärsschutz und Mitbestimmung siehe auch Jackson 2005: 275). Mitbestimmung und aktionärsorientierte Unternehmensführung scheinen 
in den als „koordiniert“ klassifizierten Ländern besser zusammenzuwirken, als man es in Kenntnis der „Varieties of Capitalism“-Diskussion erwartet hätte: it might work in practice, but it will never work in theory. Somit erscheinen auch die politischen Strategien der Mitte-Links-Spektren in den betrachteten Ländern nicht inkohärent.

Andererseits soll aber nicht ausgeschlossen werden, dass Gewerkschaften und MitteLinks-Parteien die Gewinne der abhängig Beschäftigten aus Konflikten um Managerherrschaft systematisch überschätzen, die Verluste aus Insider-Outsider-Konflikten und Klassenkonflikten hingegen systematisch unterschätzen. So ist beispielsweise zu konstatieren, dass die Einflusskanäle der deutschen Mitbestimmung durch die mit den aktionärsorientierten Reformen einhergegangene zunehmende Transparenz und Stärkung der internen Unternehmensaufsicht in der Tat vergrößert, zumindest aber gegenüber dem traditionellen Arrangement nicht verkleinert wurden. Gleichzeitig aber machen diese Reformen die Unternehmen anfälliger für den Renditedruck der Kapitalmärkte, was langfristig einen Umverteilungsprozess zu Lasten des Faktors Arbeit im Sinne de Jongs (1997) in Gang setzen muss. Deshalb ist es unseres Erachtens eine gänzlich offene Frage, als wie stabil sich die impliziten Koalitionen zwischen Minderheitsaktionären und Beschäftigten, politisch übersetzt in Vorlieben des Mitte-Links-Spektrums für aktionärsorientierte Reformen, in Zukunft erweisen werden. Die deutsche „HeuschreckenDebatte" des Jahres 2005 entstand aus Reflexion über die nachteiligen Wirkungen aktionärsorientierter Reformen für die Wählerklientel des Mitte-Links-Spektrums (ohne allerdings eine sichtbare Konsequenz für policies nach sich gezogen zu haben). Möglicherweise transformiert sich der Konflikt um Managerherrschaft langfristig in einen Insider-Outsider-Konflikt, womit aktionärsorientierte Reformen die Unterstützung des Arbeitnehmerspektrums einbüßen würden.

Das ändert allerdings nichts an den Tatsachen. Die Unternehmenskontrollsysteme westlicher Industrieländer waren bis in die 1990er Jahre hinein von Mechanismen zur Begrenzung des Aktionärseinflusses geprägt, die die institutionellen Grundlagen für den US-amerikanischen Managerialismus, den koordinierten Kapitalismus deutschen Typs, den Staatskapitalismus französischer Prägung und den italienischen Familienkapitalismus bildeten. In den 1990er Jahren setzen nachhaltige Gegenbewegungen ein, die zur systematischen Stärkung des Aktionärseinflusses führten. Bis heute gilt: Die institutionelle Transformation der Kapitalismen in Richtung eines Finanzmarktkapitalismus beruht auf politischen Koalitionen unter Einschluss des Faktors Arbeit und erfährt politische Unterstützung vor allem aus dem Mitte-Links-Spektrum.

\section{Literatur}

Aganin, Alexander/Volpin, Paolo, 2003: History of Corporate Ownership in Italy. Brüssel.

Albert, Michel, 1993: Capitalism vs. Capitalism: How America's Obsession with Individual Achievement and Short-term Profit has Led it to the Brink of Collapse. New York.

Angelides, Phil, 2005: The Right's Attack on Public Pensions, in: Los Angeles Times vom 7.2.2005, 11.

Aoki, Masahiko, 1994: The Contingent Governance of Teams: Analysis of Institutional Complementarity, in: International Economic Review 35, 657-676.

Aron, Raymond, 1964: Die industrielle Gesellschaft. 18 Vorlesungen. Frankfurt a.M. 
Berle, Adolph A./Means, Gardiner C., 1932/1999: The Modern Corporation and Private Property. With a New Introduction by Murray L. Weidenbaum and Mark Jensen. New Brunswick/London.

Beyer, Jürgen, 1998: Managerherrschaft in Deutschland? „Corporate Governance“ unter Verflechtungsbedingungen. Opladen.

Bianchi, Marcello/Bianco, Magda/Enriques, Luca, 2001: Pyramidal Groups and the Separation between Ownership and Control in Italy, in: Barca, Fabrizio/Becht, Marco (Hrsg.), The Control of Corporate Europe. Oxford, 154-187.

Bianco, Magda, 2001: Italy, in: Gugler, Klaus (Hrsg.), Corporate Governance and Economic Performance. Oxford, 130-138.

Bolt, Marie, 2000: Stellungnahme des DGB zum Fragenkatalog der Regierungskommission „Corporate Governance - Unternehmensführung - Unternehmenskontrolle - Modernisierung des Aktienrechts". Berlin.

Burnham, James, 1941: The Managerial Revolution. What is Happening in the World. Westport, Connecticut.

Burris, Val/Salt, James, 1990: The Politics of Capitalist Class Segments: A Test of Corporate Liberalism Theory, in: Social Problems 37, 341-359.

Busch, Andreas, 2003: Staat und Globalisierung. Das Politikfeld Bankenregulierung im internationalen Vergleich. Opladen.

Callaghan, Helen/Höpner, Martin, 2005: European Integration and the Clash of Capitalisms. Political Cleavages over Takeover Liberalization, in: Comparative European Politics 3, 307-332.

Chandler, Alfred D. Jr., 1977: The Visible Hand: The Managerial Revolution in American Business. Cambridge, MA.

Cioffi, John W., 2000: State of the Art: A Review Essay on Comparative Corporate Governance. The State of the Art and Emerging Research, in: American Journal of Comparative Law 48, 501534.

Cioffi, John W., 2005: Governing Globalization? The State, Law, and Structural Change in Corporate Governance, in: Clarke, Thomas (Hrsg.), Corporate Governance. Critical Perspectives on Business and Management. Volume V: Contemporary Corporate Governance. London/New York, 107-136.

Cunningham, Lawrence A., 2003: The Sarbanes-Oxley Yawn: Heavy Rhetoric, Light Reform (And it Might Just Work), in: University of Connecticut Law Review 35, 915-998.

Dalton, Russell J., 2002: Citizen Politics. Public Opinion and Political Parties in Advanced Industrial Democracies. New York/London.

Deeg, Richard, 2005: Change from Within: German and Italian Finance in the 1990s, in: Streeck, Wolfgang/Thelen, Kathleen (Hrsg.), Beyond Continuity. Institutional Change in Advanced Political Economies. Oxford, 169-202.

de Jong, Henk Wouter, 1997: The Governance Structure and Performance of Large European Corporations, in: Journal of Management and Governance 1, 5-27.

Draghi, Mario, 1998: Corporate Governance and Competitiveness, in: Review of Economic Conditions in Italy 51, 341-357.

Dyck, Alexander/Zingales, Luigi, 2002: Private Benefits of Control: An International Comparison. Unpublished Manuscript. Cambridge, MA.

Dyson, Kenneth, 1986: The State, Banks and Industry: The West German Case, in: Cox, Andrew (Hrsg.), State, Finance and Industry: A Comparative Analysis of Post-War Trends in Six Advanced Industry Economies. Brighton, Sussex, 118-141.

Fanto, James A., 1998: The Role of Corporate Law in French Corporate Governance, in: Cornell International Law Journal 31, 31-91.

Galbraith, John Kenneth, 1967: Die moderne Industriegesellschaft. München.

Goldman, Leslie, 1992: The Modernization of the French Securities Markets: Making the EEC Connection, in: Fordham Law Review 60, 227-253.

Gourevitch, Peter A./Shinn, James, 2005: Political Power and Corporate Control. The New Global Politics of Corporate Governance. Princeton/Oxford. 
Hall, Peter A., 2005: Preference Formation as a Political Process: The Case of European Monetary Union, in: Katznelson, Ira/Weingast, Barry (Hrsg.), Preferences and Situations: Points of Intersection between Historical and Rational Choice Institutionalism. London, 129-160.

Hall, Peter A./Gingerich, Daniel W., 2004: Varieties of Capitalism and Institutional Complementarities in the Political Economy: An Empirical Analysis. Köln: MPIfG Discussion Paper 2004/5.

Hall, Peter A./Soskice, David, 2001: An Introduction to Varieties of Capitalism, in: Hall, Peter A./ Soskice, David (Hrsg.), Varieties of Capitalism. Institutional Foundations of Comparative Advantage. Oxford, 1-68.

Hilferding, Rudolf, 1909/1923: Das Finanzkapital. Wien.

Hilferding, Rudolf, 1927/1982: Die Aufgaben der Sozialdemokratie in der Republik, in: Stephan, Cora (Hrsg.), Zwischen den Stühlen oder die Unvereinbarkeit von Theorie und Praxis. Schriften Rudolf Hilferdings 1904 bis 1940. Berlin/Bonn, 237-267.

Höpner, Martin, 2001: Corporate Governance in Transition: Ten Empirical Findings on Shareholder Value and Industrial Relations in Germany. Köln: MPIfG Discussion Paper 2001/5.

Höpner, Martin, 2003: Wer beherrscht die Unternehmen? Shareholder Value, Managerherrschaft und Mitbestimmung in Deutschland. Frankfurt a.M./New York.

Höpner, Martin, 2005: Sozialdemokratie, Gewerkschaften und organisierter Kapitalismus, in: Windolf, Paul (Hrsg.), Finanzmarkt-Kapitalismus. Analysen zum Wandel von Produktionsregimen. Sonderheft 45 der Kölner Zeitschrift für Soziologie und Sozialpsychologie. Wiesbaden, 196 221.

Huber, Evelyne/Ragin, Charles/Stephens, John D., 1997/2004: Comparative Welfare States Data Set 1997. Updated by David Brady, Jason Beckfield, John Stephens.

Jackson, Gregory, 2005: Employee Representation in the Board Compared: A Fuzzy Sets Analysis of Corporate Governance, Unionism and Political Institutions, in: Industrielle Beziehungen 12, 252-279.

Jacoby, Sanford M., 2005: Corporate Governance and Employees in the United States, in: Gospel, Howard/Pendleton, Andrew (Hrsg.), Corporate Governance and Labour Management. An International Comparison. Oxford/New York, 33-58.

Jensen, Michael C.IMeckling, William H., 1976: Theory of the Firm: Managerial Behavior, Agency Costs and Ownership Structure, in: Journal of Financial Economics 3, 305-360.

Judis, John B./Teixeira, Ruy, 2002: The Emerging Democratic Majority. New York/London/Toronto/ Sidney.

Kitschelt, Herbert, 1994: The Transformation of European Social Democracy. Cambridge, MA.

Kreppel, Amie, 1997: The Impact of Parties in Government on Legislative Output in Italy, in: European Journal of Political Research 31, 327-350.

Küller, Hans-Detlev, 1997: Das Shareholder-Value-Konzept aus Gewerkschaftssicht, in: Betriebwirtschaftliche Forschung und Praxis 49, 517-531.

Labaton, Stephen/Clymer, Adam, 2002: Party Lines Begin to Emerge as Congress Debates Enron, in: New York Times vom 21.3.2002, 6.

Levy, Jonah D., 1999: Tocqueville's Revenge: State, Society, and Economy in Contemporary France. Harvard University Press.

Lijphart, Arend, 1999: Patterns of Democracy. Government Forms and Performance in Thirty-Six Countries. New Haven/London.

Lütz, Susanne, 2002: Der Staat und die Globalisierung von Finanzmärkten. Regulative Politik in Deutschland, Großbritannien und den USA. Frankfurt a.M./New York.

Melis, Andrea, 2000: Corporate Governance in Italy, in: Corporate Governance: An International Review 8, 347-355.

Morin, François, 2000: A Transformation in the French Model of Shareholding and Management, in: Economy and Society 29, 36-53.

Naphtali, Fritz, 1928/1967: Wirtschaftsdemokratie. Ihr Wesen, Weg und Ziel. Mit einem Vorwort von Ludwig Rosenberg und einer Einführung von Otto Brenner. Frankfurt a.M.

Neue Zürcher Zeitung, 2002: Wenig Aktionärsdemokratie in Italien, in: NZZ vom 1.2.2002, 31.

O'Sullivan, Mary, 2001: Change and Continuity in the French System of Corporate Governance. Fontainebleau Cedex. 
Pagano, Ugo/Trento, Sandro, 2002: Continuity and Change in Italian Corporate Governance: The Institutional Stability of one Variety of Capitalism. Siena.

Piazza, James, 2001: De-linking Labor - Labor Unions and Social Democratic Parties under Globalization, in: Party Politics 7, 413-435.

Rappaport, Alfred, 1986/1999: Shareholder Value. Ein Handbuch für Manager und Investoren. Übersetzt von Wolfgang Klien. 2., vollständig überarbeitete und aktualisierte Auflage. Stuttgart.

Roe, Mark J., 1994: Strong Managers, Weak Owners. The Political Roots of American Corporate Finance. Princeton.

Roe, Mark J., 2003: Political Determinants of Corporate Governance. Political Context, Corporate Impact. Oxford.

Ross, Fiona, 2000: „Beyond left and right“: The New Partisan Politics of Welfare, in: Governance: An International Journal of Policy and Administration 13, 155-183.

Scheibe-Lange, Ingrid/Prangenberg, Arno, 1997: Mehr Mitbestimmung via US-Börsenaufsicht? in: Die Mitbestimmung 43, 45-49.

Schmidt, Manfred G., 1996: When Parties Matter: A Review of the Possibilities and Limits of Partisan Influence on Public Policy, in: European Journal of Political Research 30, 155-183.

Schmidt, Manfred G., 2000: Demokratietheorien. Eine Einführung. 3., überarbeitete und erweiterte Auflage. Opladen.

Schmidt, Manfred G., 2002: The Impact of Political Parties, Constitutional Structures and Veto Players on Public Policy, in: Keman, Hans (Hrsg.), Comparative Democratic Politics. A Guide to Contemporary Theory and Research. London, 166-184.

Schumpeter, Joseph A., 1942: Kapitalismus, Sozialismus und Demokratie. Einleitung von Edgar Salin. Bern.

Shleifer, Andrei/Summers, Lawrence H., 1988: Breach of Trust in Hostile Takeovers, in: Auerbach, Alan J. (Hrsg.), Corporate Takeovers: Causes and Consequences. Chicago, 33-68.

Shonfield, Andrew, 1965: Modern Capitalism. The Changing Balance of Public and Private Power. Oxford.

Stevenson, Richard W./Oppel, Richard A., 2002: Democrats Pushing Bush to Act on Business Fraud, in: New York Times vom 2.7.2002, 8.

Streeck, Wolfgang, 2001: Introduction: Explorations into the Origins of Nonliberal Capitalism in Germany and Japan, in: Streeck, Wolfgang/Yamamura, Kozo (Hrsg.), The Origins of Nonliberal Capitalism: Germany and Japan. Ithaca/London, 1-38.

The Economist, 2001: A Survey of Italy. Beilage zum Economist vom 7.7.2001.

Tiberghien, Yves, 2006: Invisible Reforms: Globalization, State Mediation, and Corporate Restructuring. Ithaca.

Tinbergen, Jan, 1961: Do Communist and Free Economies Show a Converging Pattern?, in: Soviet Studies 8, 333-341.

Ulmer, Peter, 1980: Die Anpassung der Satzungen mitbestimmter Aktiengesellschaften an das MitbestG 1976. Heidelberg.

Wilensky, Harold, 2002: Rich Democracies. Political Economy, Public Policy, and Performance. Berkeley.

Windolf, Paul, 2005: Was ist Finanzmarkt-Kapitalismus?, in: Windolf, Paul (Hrsg.), FinanzmarktKapitalismus. Analysen zum Wandel von Produktionsregimen. Sonderheft 45 der Kölner Zeitschrift für Soziologie und Sozialpsychologie. Wiesbaden, 20-57.

Windolf, Paul/Beyer, Jürgen, 1995: Kooperativer Kapitalismus. Unternehmensverflechtungen im internationalen Vergleich, in: Kölner Zeitschrift für Soziologie und Sozialpsychologie 47, 1-36.

Winkler, Heinrich August, 1974: Organisierter Kapitalismus. Voraussetzungen und Anfänge. Göttingen.

Zohlnhöfer, Reimut, 2001: Die Wirtschaftspolitik der Ära Kohl. Eine Analyse der Schlüsselentscheidungen in den Politikfeldern Finanzen, Arbeit und Entstaatlichung, 1982-1998. Opladen.

Zysman, John, 1983: Governments, Markets, and Growth: Financial Systems and the Politics of Industrial Change. Ithaca/London. 